\title{
Liquid-bridge stability and breakup on surfaces with contact-angle hysteresis
}

\author{
Amir Akbari and Reghan J. Hill* \\ Department of Chemical Engineering, McGill University, 3610 \\ University Street, Montreal, Quebec, H3A 0C5
}

\begin{abstract}
We study the stability and breakup of liquid bridges with a free contact line on surfaces with contact-angle hysteresis (CAH) under zero-gravity conditions. Non-ideal surfaces exhibit $\mathrm{CAH}$ because of surface imperfections, by which the constraints on three-phase contact lines are influenced. Given that interfacial instabilities are constraintsensitive, understanding how $\mathrm{CAH}$ affects the stability and breakup of liquid bridges is crucial for predicting the drop size in contact-drop dispensing. Unlike ideal surfaces on which contact lines are always free irrespective of surface wettability, contact lines may undergo transitions from pinned to free and vice-versa during drop deposition on non-ideal surfaces. Here, we experimentally and theoretically examine how stability and breakup are affected by $\mathrm{CAH}$, highlighting cases where stability is lost during a transition from a pinned-pinned (more constrained) to pinned-free (less constrained) interface - rather than a critical state. This provides a practical means of expediting or delaying stability loss. We also demonstrate how the dynamic contact angle can control the contact-line radius following stability loss.
\end{abstract}

*E-mail: reghan.hill@mcgill.ca 


\section{Introduction}

Studying the stability and dynamics of liquid bridges is motivated by a broad range of applications, including crystal growth in microgravity ${ }^{1}$, surface patterning, nano-printing, and nano-lithography ${ }^{2-4}$, aggregation and coalescence of flexible fibres ${ }^{5-8}$, and capillary induced collapse of elastic structures ${ }^{9-14}$. Quantifying liquid-bridge and jet breakup upon stability loss dates to the works of Plateau ${ }^{15}$ and Rayleigh ${ }^{16},{ }^{17}$. While early investigations of crystal growth and purification focused on determining the minimum liquid volume that can be held between circular discs ${ }^{18,19}$, the drop-size distribution following breakup is of prime interest in contact-drop dispensing and liquid-transfer applications $^{20-22}$. Minimizing the dispensed-drop size relative to the needle diameter is central to surface patterning based on direct-write lithographic techniques ${ }^{3,23}$.

Recent studies of contact-drop dispensing have shown that the deposited drop size is influenced by the needle retraction speed, needle-tip size, surface characteristics, and dispensing control parameters ${ }^{24,25}$. Interestingly, the deposited drop volume in pressure-controlled and volume-controlled dispensing behave differently with the needle retraction speed. Faster retraction reduces the drop size to a minimum, and monotonically increases the drop size in pressure-controlled and volume-controlled dispensing, respectively. Three regimes were experimentally identified with respect to the retraction speed $U_{n}$ for the pressure-controlled case. In the first two, $U_{n} \ll u_{w}$, where $u_{w}$ is the capillary-wave speed; the contact line advances in the first and is stationary in the second with the drop size scaling as $U_{n}^{-1 / 2}$; the third corresponds to fast retraction speeds, $U_{n} / u_{w} \sim O\left(10^{-2}\right)$, where the dynamics dramatically change, and the drop size does not scale with $U_{n}$ as a simple power law. Here, the drop size is almost two orders of magnitude smaller than in the first two regimes, which Qian et al. ${ }^{24}$ attributed to a fast receding contact line with a speed approaching $u_{w}$. However, in volume-controlled deposition, the dispensed-drop size did not exhibit the same sensitivity to the needle retraction speed in the parameter range studied by Qian and Breuer ${ }^{25}$. Thus, our study is motivated, in part, by seeking to answer whether it is possible to influence - by purely geometric means - the dynamics in volume-controlled deposition, so that one may achieve comparable sensitivity as in the pressurecontrolled case, to achieve small-drop deposition.

Experimental studies of static liquid bridges between equal circular discs are extensive. Using neutral-buoyancy experiments, Sanz and Martinez ${ }^{26}$ 
ascertained the minimum-volume stability limit ${ }^{1}$ in the slenderness range $0<\Lambda<6$. Russo and Steen ${ }^{27}$ determined the maximum-volume stability limit in a similar set-up, showing that axisymmetric liquid bridges nonaxisymmetrically bulge when their interface is tangent to the discs. The experiments of Slobozhanin et al. ${ }^{28}$ provided further insights on this stability limit. They showed that above (below) the slenderness $\Lambda \simeq 0.4946$, liquid bridges continuously (abruptly) bulge into a non-axisymmetric shape. Other studies considered the effect of gravity on the stability limits of axisymmetric ${ }^{29}$ and non-axisymmetric liquid bridges ${ }^{30}$ between equal discs. Emphasizing the destabilizing effect of gravity on nearly cylindrical liquid bridges, Lowry and Steen ${ }^{31}$ experimentally demonstrated that subjecting liquid bridges to an external laminar flow suppresses interfacial disturbances, thereby stretching the stability limit beyond that of static bridges.

Surface imperfections (e.g., heterogeneity and roughness) complicate the equilibrium of gas-liquid-solid contact lines on non-ideal surfaces ${ }^{25,32,33}$. Contact lines remain pinned on non-ideal surfaces as long as the equilibrium contact angle is between the receding and advancing contact angles, and are mobile otherwise ${ }^{34}$. The receding contact angle particularly plays a key role in contact-drop dispensing when the contact line is free ${ }^{25,35}$. Chen et al. ${ }^{33},{ }^{36}$ experimentally and numerically studied the effect of contact-angle hysteresis $(\mathrm{CAH})$ on the evolution and adhesion force of liquid bridges with two free contact lines. Similarly to Qian and Breuer ${ }^{25}$, experiments were conducted in a liquid-gas system where the gravity effect is alleviated by small bridge dimensions. Using similar experiments, Chen et al. ${ }^{22}$ examined bridge breakup, showing that the liquid transfer ratio is correlated with the difference between the receding contact angles on the plates.

Dynamic effects, manifesting in the capillary number $\mathrm{Ca}$ and Reynolds number Re, are not negligible at large $U_{n}$, and should be examined in liquidtransfer applications. Dodds et al. ${ }^{20}$, Chen et al. ${ }^{37}$ and Chen et al. ${ }^{38}$ have studied the breakup of liquid bridges with free contact lines between two supports with respect to $U_{n}$. Depending on the magnitude of $U_{n}$, two distinct regimes were identified: (i) $\mathrm{Ca} \gtrsim O(1), \mathrm{Re} \ll 1$ and (ii) $\mathrm{Ca}, \mathrm{Re} \gtrsim O(1)$. In the first, liquid bridges asymptotically tended to more evenly break between the supports with increasing $U_{n}$, almost irrespective of the support wetta-

\footnotetext{
${ }^{1}$ The maximum-volume stability limit at fixed slenderness, referred to as the 'rotund limit', is equivalent to the minimum-slenderness stability limit at fixed volume. Similarly, the minimum-volume stability limit at fixed slenderness, referred to as the 'slender limit', is equivalent to the maximum-slenderness stability limit at fixed volume.
} 
bility. This restriction limits the minimum dispensed-drop size that can be achieved by increasing the retraction speed. In contrast, the dispensed-drop size monotonically decreased with increasing $U_{n}$ in the range of $\mathrm{Ca}$ and Re studied by Chen et al. ${ }^{38}$. Nevertheless, CAH effects on the stability and dynamics of liquid bridges in contact-drop dispensing have not been fully understood in the literature.

The advancing and receding contact angles reflect surface characteristics and differences between non-ideal and ideal surfaces. However, they are not constant thermodynamic quantities and may vary on heterogeneous surfaces according to the contact-line position. Variable contact angles are also observed when dispensing a liquid onto a surface where there is strong adhesion. Here, a moving contact line can deposit material on the surface as it recedes, changing interfacial tensions and the receding contact angle. This 'surface contamination' effect must be accounted for in conventional contactangle-hysteresis experiments where liquid bridges are squeezed and stretched sequentially on the same substrate.

Depending on the surface wettability and drop volume, the contact angle may be equal to or between receding and advancing contact angles when stretching liquid bridges. Hence, a bridge can undergo transitions from pinned-pinned to pinned-free contact lines (and vice versa) during stretching. These complications raise new, non-trivial questions as to how the stability limits and dispensed-drop volume are influenced by $\mathrm{CAH}$, and which stability limit (with respect to pinned-pinned or pinned-free disturbances) determines the breakup height.

In this paper, we address the forgoing questions by studying pinnedpinned to pinned-free transitions and their respective stability limits during drop deposition on surfaces with CAH. In particular, we show that, contrary to the common notion, there are cases where liquid bridges do not break at a critical state (i.e., the pinned-pinned or pinned-free stability limit). To the best of our knowledge, this has not been previously reported in the literature. Moreover, we experimentally verify the theoretical predictions of the maximum- and minimum-slenderness stability limits ${ }^{35}$ when the contact line is free at breakup. The stability limits of liquid bridges with pinned and free contact lines are compared to demonstrate the destabilizing effect of free contact lines. To simulate zero gravity using the density matching technique, liquid bridges of silicon oil are formed in a water-methanol solution. The contact-angle effect is then examined by adding surfactant to the aqueous phase. 


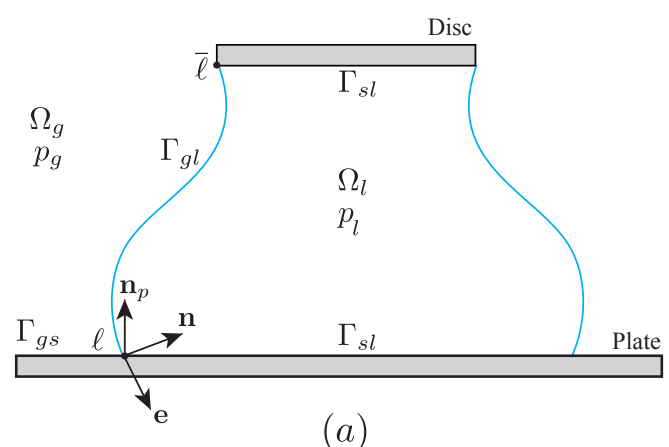

(a)

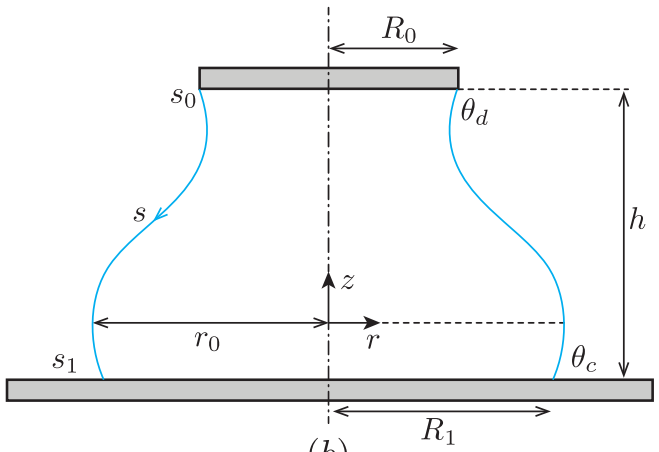

(b)

Figure 1: Weightless liquid bridge; (a) schematic and (b) coordinate system with meridian curve parametrization.

\section{Theory}

Consider a liquid of volume $v$ bridging a circular disk with radius $R_{0}$ and a large plate. The disc and plate are separated by a distance $h$, as shown in Fig. 1. The bridge is pinned to the disc and is free to slide horizontally on the plate. A small Bond number $(\mathrm{Bo} \ll 1)$ is achieved by density matching, so the gravity force is negligible. Consequently, there is a constant pressure differential between the non-hydrostatic pressure of the bridge $p_{l}$ and the surrounding fluid $p_{g}$. Here, the surface tension between the phases $i$ and $j$ is denoted $\gamma_{i j}$ with $\Gamma_{i j}$ the corresponding interfacial surface area. The contact and dihedral angles that the interface $\Gamma_{g l}$ forms with the plate and disc are denoted $\theta_{c}$ and $\theta_{d}$, respectively. The cylindrical volume $V=v /\left(\pi R_{0}^{2} h\right)$, scaled volume $v^{*}=v /\left(4 \pi R_{0}^{3} / 3\right)$, scaled pressure (mean curvature) $Q=q R_{0}$, and slenderness $\Lambda=h / R_{0}$ are the dimensionless parameters with which the liquid bridges are specified. Note that $q=\left(p_{g}-p_{l}\right) / \gamma_{g l}$ measures the nonhydrostatic pressure differential ${ }^{39}$.

We adopt $\theta_{c}$ to denote the angle between the tangent to the bridge surface at the contact line $\ell$ and plate. This assumes the following values: (i) a constant when the surface is ideal, (ii) the advancing or receding contact angle when the surface is non-ideal, or (ii) a geometric parameter (similarly to $\theta_{d}$ ) when the contact line is pinned. The receding and advancing contact angles are specifically identified by $\theta_{a}$ and $\theta_{r}$, respectively; on heterogeneous surfaces, these vary with the contact-line position. Note that these angles, as defined in Fig. 1, are supplementary to their conventional definition ${ }^{40}$ where 
they are measured through the liquid bridge. According to our definition, $\theta_{a}<\theta_{r}$, implying that $\theta_{r}$ and $\theta_{a}$ are, respectively, the upper and lower bounds on $\theta_{c}$ for which the contact line remains pinned.

Stable equilibria of the system in Fig. 1 are determined by minimizing the total potential energy

$$
E=\gamma_{s l} \Gamma_{s l}+\gamma_{g l} \Gamma_{g l}+\gamma_{s g} \Gamma_{s g}
$$

with volume $v=$ const. Parametrizing axisymmetric meridian curves by $t$, which varies in the fixed interval $\left[t_{0}, t_{1}\right]$ in equilibrium and perturbed states such that $t_{0}$ and $t_{1}$, respectively, correspond to the contact lines $\bar{\ell}$ and $\ell$ (see Fig. 1), $E$ is given by the functional

$$
E[r(t), z(t)]=\gamma_{g l} \int_{t_{0}}^{t_{1}} \Phi\left(r, z, r^{\prime}, z^{\prime}\right) \mathrm{d} t+\frac{R_{1}}{2}\left(\gamma_{s l}-\gamma_{s g}\right)
$$

where $\mathbf{r}(t)=r(t) \mathbf{e}_{r}+z(t) \mathbf{e}_{z}$ specifies the meridian curves, $\Phi\left(r, z, r^{\prime}, z^{\prime}\right)=$ $r \sqrt{r^{\prime 2}+z^{\prime 2}}+\lambda r^{2} z^{\prime}$, and $\lambda$ is the Lagrange multiplier associated with the volume constraint $v=\pi \int_{t_{0}}^{t_{1}} r^{2} z^{\prime} \mathrm{d} t=$ const. General axisymmetric variations of a meridian curve with respect its equilibrium state $\hat{\mathbf{r}}(t)=\hat{r}(t) \mathbf{e}_{r}+\hat{z}(t) \mathbf{e}_{z}$ can be written

$$
\begin{aligned}
& z(t)=\hat{z}(t)+\eta_{1}(t) \epsilon+\eta_{2}(t) \epsilon^{2}+\cdots, \\
& r(t)=\hat{r}(t)+\psi_{1}(t) \epsilon+\psi_{2}(t) \epsilon^{2}+\cdots
\end{aligned}
$$

with

$$
\begin{gathered}
\eta_{1}\left(t_{0}\right), \eta_{2}\left(t_{0}\right)=0 \quad \eta_{1}\left(t_{1}\right), \eta_{2}\left(t_{1}\right)=0, \\
\psi_{1}\left(t_{0}\right), \psi_{2}\left(t_{0}\right)=0 \quad \psi_{1}\left(t_{1}\right), \psi_{2}\left(t_{1}\right)=\text { finite }
\end{gathered}
$$

which upon substitution in Eq. (2) furnish the first variation

$$
\begin{array}{r}
\frac{\delta E}{\epsilon}=\gamma_{g l} \int_{t_{0}}^{t_{1}}\left[\Phi_{r}+\frac{\mathrm{d}}{\mathrm{d} t} \Phi_{r^{\prime}}\right] \\
\psi_{1} \mathrm{~d} t+\gamma_{g l} \int_{t_{0}}^{t_{1}}\left[\Phi_{z}+\frac{\mathrm{d}}{\mathrm{d} t} \Phi_{z^{\prime}}\right] \eta_{1} \mathrm{~d} t \\
+\left[\left.\gamma_{g l} \Phi_{r^{\prime}}\right|_{t_{1}}+R_{1}\left(\gamma_{s l}-\gamma_{s g}\right)\right] \psi_{1}\left(t_{1}\right) .
\end{array}
$$

Since the contact line $\ell$ is free, $\Gamma_{g l}$ admits all perturbations satisfying Eqs. (5) and (6), including those with $\psi_{1}\left(t_{1}\right)=0$ and $\psi_{1}\left(t_{1}\right) \neq 0$. Equilibrium states are those for which $\delta E=0$ with respect to admissible perturbations $\eta_{1}(t)$ and $\psi_{1}(t)$. Hence, all the expressions enclosed by square brackets in Eq. (7) must vanish. Since $z(t)$ and $r(t)$ represent a single planar curve, $\Phi_{r}+\mathrm{d} \Phi_{r^{\prime}} / \mathrm{d} t=0$ 
and $\Phi_{z}+\mathrm{d} \Phi_{z^{\prime}} / \mathrm{d} t=0$ are not independent ${ }^{41}$, and both lead to the same equilibrium condition

$$
\frac{r^{\prime \prime} z^{\prime}-r^{\prime} z^{\prime \prime}}{\left(r^{\prime 2}+z^{\prime 2}\right)^{3 / 2}}-\frac{z^{\prime}}{r\left(r^{\prime 2}+z^{\prime 2}\right)^{1 / 2}}=2 \lambda
$$

Comparing the potential energy in Eq. (1) with the total grand canonical potential, the Lagrange multiplier is determined $\lambda=\left(p_{l}-p_{g}\right) / 2 \gamma_{g l}=-q / 2$ (see Akbari et al. ${ }^{14}$ and Neumann et al. ${ }^{42}$ ). In general, $\psi_{1}\left(t_{1}\right) \neq 0$, so equilibrium requires $\left.\gamma_{g l} \Phi_{r^{\prime}}\right|_{t_{1}}+R_{1}\left(\gamma_{s l}-\gamma_{s g}\right)=0$, furnishing the contact-line constraint

$$
\gamma_{g l} \cos \theta_{c}=\gamma_{s g}-\gamma_{s l} \quad \text { with } \quad \cos \theta_{c}=\mathbf{n} \cdot \mathbf{n}_{p}
$$

If $\ell$ were a pinned contact line, admissible perturbations would have to satisfy $\psi_{1}\left(t_{1}\right)=0$, so the forgoing boundary term could take any arbitrary value without affecting the first variation; this explains why equilibrium imposes no restriction on $\theta_{c}$ at pinned contact lines. Parametrizing meridian curves by their arclength (i.e., $t=s$ ) and introducing the scaled lengths

$$
\rho=|q| r, \quad \xi=q z, \quad \tau=|q| s
$$

reduces Eq. (8) to

$$
\rho^{\prime} \xi^{\prime \prime}-\rho^{\prime \prime} \xi^{\prime}=1-\frac{\xi^{\prime}}{\rho}
$$

Using $\rho^{\prime 2}+\xi^{\prime 2}=1$ with its equivalent representation $\rho^{\prime} \rho^{\prime \prime}+\xi^{\prime} \xi^{\prime \prime}=0$, and solving Eq. (11) for $\rho^{\prime \prime}$ and $\xi^{\prime \prime}$ furnishes the parametric, axisymmetric form of the Young-Laplace equation

$$
\left\{\begin{array}{l}
\rho^{\prime \prime}=-\xi^{\prime}\left(1-\xi^{\prime} / \rho\right) \\
\xi^{\prime \prime}=\rho^{\prime}\left(1-\xi^{\prime} / \rho\right)
\end{array} \quad\left({ }^{\prime} \equiv \mathrm{d} / \mathrm{d} \tau\right),\right.
$$

which can be found elsewhere ${ }^{35,39}$.

We apply the variational method of Myshkis et al. ${ }^{39}$ to determine the stability of equilibrium surfaces with respect to arbitrary volume-preserving perturbations. This method associates the second variation of the potential energy with the eigenvalues of the corresponding Sturm-Liouville problem where critical states satisfy

$$
\left\{\begin{array}{l}
\mathscr{L} \varphi_{0}+\mu=0 \\
\varphi_{0}\left(\tau_{0}\right)=0, \\
\int_{\tau_{0}}^{\tau_{1}} \rho \varphi_{0} \mathrm{~d} \tau=0
\end{array} \varphi_{0}^{\prime}\left(\tau_{1}\right)+\tilde{\chi} \varphi_{0}\left(\tau_{1}\right)=0\right.
$$


for axisymmetric perturbations and

$$
\left\{\begin{array}{l}
\left(\mathscr{L}-1 / \rho^{2}\right) \varphi_{1}=0 \\
\varphi_{1}\left(\tau_{0}\right)=0, \quad \varphi_{1}^{\prime}\left(\tau_{1}\right)+\tilde{\chi} \varphi_{1}\left(\tau_{1}\right)=0
\end{array}\right.
$$

for non-axisymmetric perturbations. Here,

$$
\chi=\frac{k_{1 \ell} \cos \theta_{c}-k_{p \ell}}{\sin \theta_{c}} \text { at } \ell,
$$

and

$$
\mathscr{L} \equiv \frac{\mathrm{d}^{2}}{\mathrm{~d} \tau^{2}}+\frac{\rho^{\prime}}{\rho} \frac{\mathrm{d}}{\mathrm{d} \tau}+\left[\left(1-\frac{\xi^{\prime}}{\rho}\right)^{2}+\left(\frac{\xi^{\prime}}{\rho}\right)^{2}\right]
$$

with $\tilde{\chi}=\chi /|q|$; the first principal curvatures of the interface and plate at $\ell$ are denoted $k_{1 \ell}$ and $k_{p \ell}$, respectively. Here, $\varphi_{0}(\tau)$ and $\varphi_{1}(\tau)$ represent axisymmetric and non-axisymmetric perturbations. The solutions of Eqs. (13) and (14) can be written

$$
\begin{gathered}
\varphi_{0}(\tau)=C_{1} w_{1}(\tau)+C_{2} w_{2}(\tau)+\mu w_{3}(\tau), \\
\varphi_{1}(\tau)=C_{4} w_{4}(\tau)+C_{5} w_{5}(\tau) .
\end{gathered}
$$

An equilibrium state is critical if $\varphi_{0}$ or $\varphi_{1}$ has a non-trivial solution. It can be shown (see chapter 3 of Myshkis et al. ${ }^{39}$ ) that a non-trivial solution for $\varphi_{0}\left(\varphi_{1}\right)$ exists provided $\tilde{\chi}=\tilde{\chi}^{0}\left(\tilde{\chi}=\tilde{\chi}^{1}\right)$, where

$$
\begin{aligned}
& \tilde{\chi}^{0}=-\frac{\left|\begin{array}{ccc}
w_{1}\left(\tau_{0}\right) & w_{2}\left(\tau_{0}\right) & w_{3}\left(\tau_{0}\right) \\
w_{1}^{\prime}\left(\tau_{1}\right) & w_{2}^{\prime}\left(\tau_{1}\right) & w_{3}^{\prime}\left(\tau_{1}\right) \\
\int_{\tau_{0}}^{\tau_{1}} \rho w_{1} \mathrm{~d} \tau & \int_{\tau_{0}}^{\tau_{1}} \rho w_{2} \mathrm{~d} \tau & \int_{\tau_{0}}^{\tau_{1}} \rho w_{3} \mathrm{~d} \tau
\end{array}\right|}{\left|\begin{array}{ccc}
w_{1}\left(\tau_{0}\right) & w_{2}\left(\tau_{0}\right) & w_{3}\left(\tau_{0}\right) \\
w_{1}\left(\tau_{1}\right) & w_{2}\left(\tau_{1}\right) & w_{3}\left(\tau_{1}\right) \\
\int_{\tau_{0}}^{\tau_{1}} \rho w_{1} \mathrm{~d} \tau & \int_{\tau_{0}}^{\tau_{1}} \rho w_{2} \mathrm{~d} \tau & \int_{\tau_{0}}^{\tau_{1}} \rho w_{3} \mathrm{~d} \tau
\end{array}\right|} \\
& \tilde{\chi}^{1}=-\frac{\left|\begin{array}{ll}
w_{4}\left(\tau_{0}\right) & w_{5}\left(\tau_{0}\right) \\
w_{4}^{\prime}\left(\tau_{1}\right) & w_{5}^{\prime}\left(\tau_{1}\right)
\end{array}\right|}{\left|\begin{array}{ll}
w_{4}\left(\tau_{0}\right) & w_{5}\left(\tau_{0}\right) \\
w_{4}\left(\tau_{1}\right) & w_{5}\left(\tau_{1}\right)
\end{array}\right|}
\end{aligned}
$$

with $\tilde{\chi}^{0}$ and $\tilde{\chi}^{1}$ the critical $\tilde{\chi}$, which, respectively, correspond to axisymmetric and non-axisymmetric perturbations. From the properties of the spectral problem, one can deduce the stability criterion

$$
\tilde{\chi}>\max \left\{\tilde{\chi}^{0}, \tilde{\chi}^{1}\right\} \text {. }
$$


Solving the Young-Laplace equation furnishes the equilibrium meridian curve

$$
\left\{\begin{array}{l}
\rho(\tau)=\sqrt{1+a^{2}+2 a \cos \tau} \\
\xi(\tau)=\int_{0}^{\tau} \frac{1+a \cos t}{\rho(t)} \mathrm{d} t
\end{array}\right.
$$

giving $^{35}$

$$
\begin{gathered}
|Q|=\sqrt{1+a^{2}+2 a \cos \tau_{0}}, \\
\Lambda=-\frac{1}{Q} \int_{\tau_{0}}^{\tau_{1}} \frac{1+a \cos t}{\rho(t)} \mathrm{d} t, \\
\tan \theta_{c}=-\operatorname{sign}(Q) \frac{1+a \cos \tau_{1}}{a \sin \tau_{1}}, \\
v^{*}=-\frac{3}{4 Q} \int_{\tau_{0}}^{\tau_{1}}(1+a \cos t) \rho(t) \mathrm{d} t,
\end{gathered}
$$

where $a=\rho(0)-1$ is a shape parameter. We solve Eqs. (23)-(26) to obtain $\left(\tau_{0}, \tau_{1}, a, \Lambda\right)$ at fixed $v^{*}$ and $\theta_{c}$ with respect to $Q$ as the branching parameter using Keller's arclength continuation method ${ }^{43}$. Stability along equilibrium branches are determined by calculating $\tilde{\chi}^{0}$ and $\tilde{\chi}^{1}$ from Eqs. (19) and (20), and examining the stability criterion Eq. (21). Note that pinned-free and pinned-pinned liquid bridges are, respectively, specified by $\left(\Lambda, V, \theta_{c}\right)$ and $(\Lambda, V, K)$, where $K=R_{1} / R_{0}$ is the ratio of the lower to upper contact-line radii. When stretching the bridge, $K$ varies at fixed $\theta_{c}$ in pinned-free bridges, and $\theta_{c}$ varies at fixed $K$ in pinned-pinned bridges.

Akbari et al. ${ }^{35}$ showed that critical surfaces at the minimum-slenderness stability limit, corresponding to the upper boundary of the stability region, are nodoids with $\theta_{d}=0$, the volume of which is well approximated by

$$
\begin{aligned}
V & =1+\frac{1}{4} \sec ^{4}\left(\theta_{c} / 2\right)\left(\pi-\theta_{c}+\cos \theta_{c} \sin \theta_{c}\right) \Lambda \\
& -\frac{1}{384} \sec ^{8}\left(\theta_{c} / 2\right)\left[-97+24\left(\pi-\theta_{c}\right)^{2}-136 \cos \theta_{c}-32 \cos \left(2 \theta_{c}\right)\right. \\
& \left.+8 \cos \left(3 \theta_{c}\right)+\cos \left(4 \theta_{c}\right)+24\left(\pi-\theta_{c}\right) \sin \left(2 \theta_{c}\right)\right] \Lambda^{2}+O\left(\Lambda^{3}\right)
\end{aligned}
$$

in $V$ versus $\Lambda$ stability diagrams. 


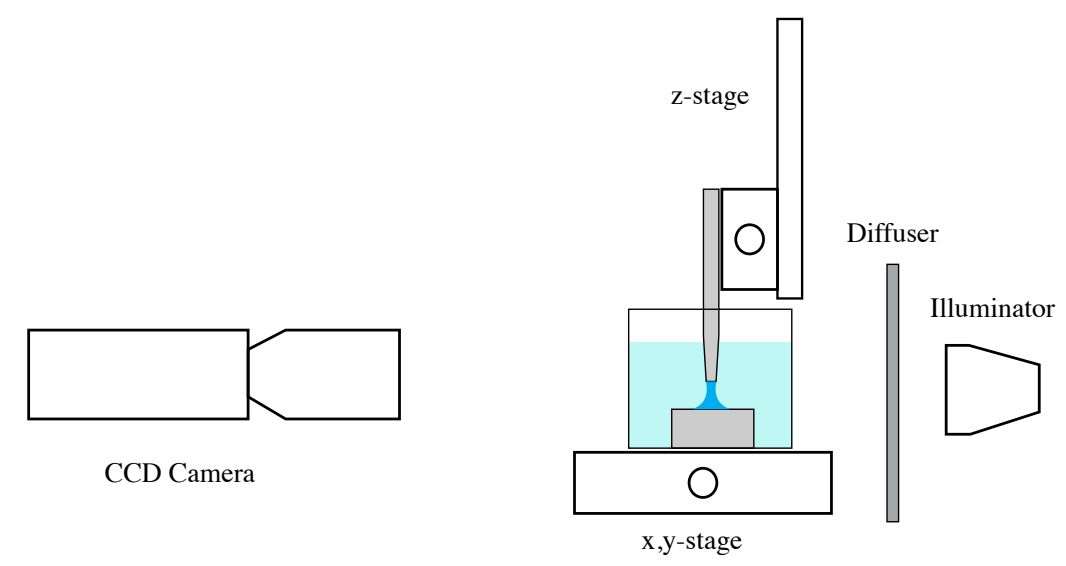

Figure 2: Schematic of the experimental setup.

\section{Materials and methods}

\subsection{Experimental set-up}

Experiments were performed in a cubic Plateau tank under neutrally buoyant conditions (Fig. 2). This mimicked micro-gravity conditions for larger menisci and, thus, enhanced the accuracy and resolution of image processing. Silicon oil ( 5 cSt, Sigma Aldrich) with specific gravity 0.92 was used as the bridge in a $100 \mathrm{ml}$ water-methanol solution (volumetric mixing ratio 42:58) bath. The composition of the bath solution was adjusted so that its density matched that of silicon oil at the experiment temperature $\left(\approx 20^{\circ} \mathrm{C}\right)$, whereby an average density difference $\Delta \rho \approx 5 \times 10^{-3} \mathrm{~g} \mathrm{~cm}^{-3}$ was achieved. Using a microsyringe, a drop with a prescribed volume in the range $5-50 \mu \mathrm{l}$ was deposited onto a plastic coverslip (Fischer Scientific), which had been soaked in a $0.1 \mathrm{M}$ hydrochloric acid solution, rinsed with DI water, and placed in the tank. A bridge was produced by gently pressing a needle with

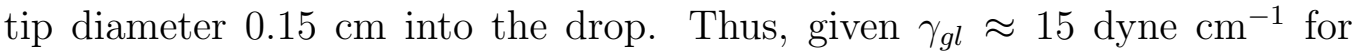
water-methanol-silicon oil system and $R_{0} \approx 0.075 \mathrm{~cm}$, the Bond number was Bo $=\Delta \rho g R_{0}^{2} / \gamma_{g l} \approx 0.002$. The needle was mounted on a one-dimensional vertical translation stage to control the bridge height, and the tank was placed on a two-dimensional positioning stage to align the drop and needle centers before contact, ensuring that the bridge is axisymmetric. The maximum (minimum) slenderness stability limit was ascertained by stretching (squeezing) the bridge until the bridge ruptured (bulged asymmetrically). 
A CCD camera (Prosilica GX1050, Allied Vision) with a $5 \times$ lens (Nikon GMicro-NIKKOR) was used to record the bridge dynamics. Images were analyzed using an in-house Matlab script. The bridge contact angle with the coverslip was adjusted by changing the interfacial tensions in the system using the anionic surfactant sodium dodecyl sulfate (SDS) (Sigma Aldrich) at concentrations in the range $0-10 \mathrm{~g} \mathrm{l}^{-1}$. Once deposited on a coverslip, silicon-oil drops were maintained in the bath for an hour before performing experiments to ensure a uniform equilibrium concentration on the interface and throughout the bath. Thus, the concentration gradient (driving force for Marangoni convection) is not expected to significantly affect the stability limits.

\subsection{Procedure}

Since the needle was hollow with a sharp-edged tip, bridges were always pinned to the needle. After a drop was deposited onto a coverslip, it was squeezed in 0.01 inch steps to reach the minimum-slenderness stability limit, at which it bulged asymmetrically; bridges were imaged at each step. The maximum-slenderness stability limit was similarly measured by stretching bridges until rupture. In the slender limit, the bridge broke into two primary drops, leaving several satellite drops suspended in the bath. Without SDS, the contact line was free at breakup only for small drop volumes (less than $10 \mu \mathrm{l}$ ), and was otherwise pinned at larger volumes. Since the emphasis in this work is on the role of moving contact lines, SDS was added to the bath to reduce the receding contact angle (see Fig. 1) of the bridge on the coverslip.

\subsection{Feature extraction}

The bridge interface with the bath solution was ascertained using a gradientbased edge detection method with a Gaussian optimal smoothing filter ${ }^{44}$. In this method, pixels on an interface are identified by finding maxima in the first directional derivative of intensity; or, equivalently, seeking zero-crossings in the second directional derivative. Derivatives were taken along normals to interfaces using high-order (8-10 points) central schemes. Then, the analytical solution of the bridge meridian curve, given by Eq. (22), was fitted to the extracted interfaces. The unknown parameters $\left(Q, \tau_{0}, \tau_{1}, a\right)$ were determined by minimizing the root-mean-squared normal distances between the extracted interface pixels and the theoretical meridian curve. Figure 3 shows 


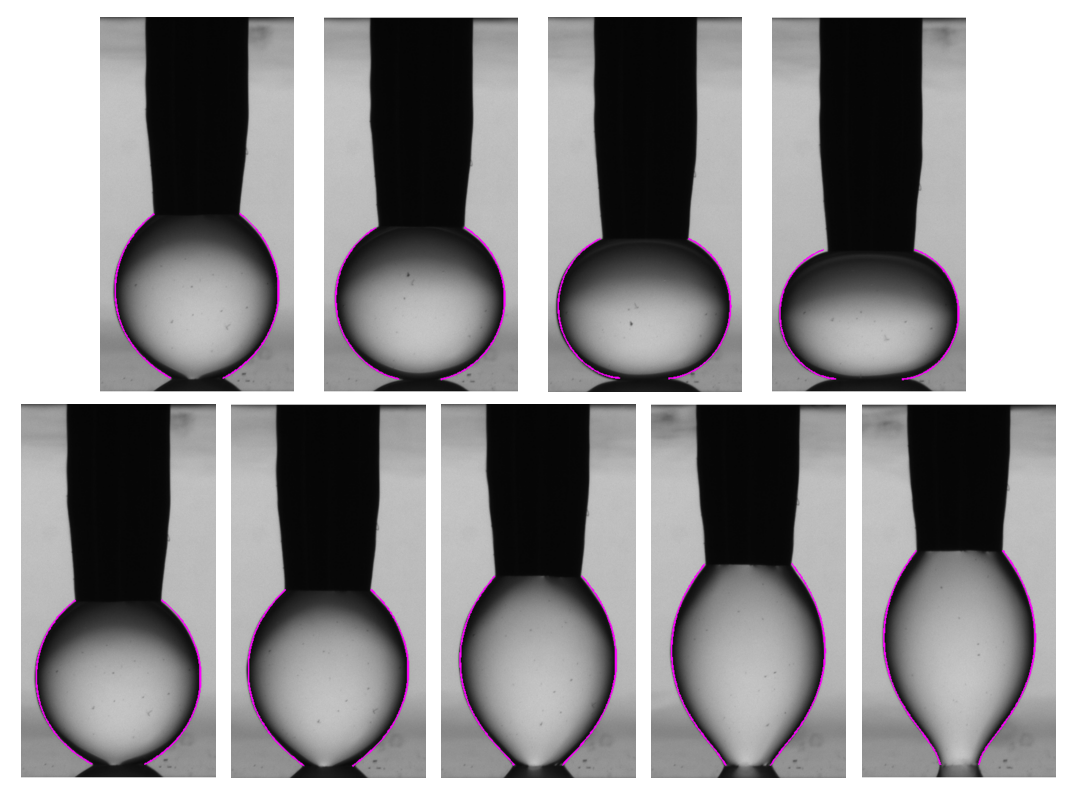

Figure 3: Fitting theoretical meridian curves to bridges boundaries in stretching (bottom) and squeezing (top) sequences (20 $\mu$ l drop).

typical results of the image-processing script for stretching and squeezing experiments.

\section{Stability-constraint relationship: Overview}

Contact-line and bulk constraints stabilize capillary surfaces by restricting the class of disturbances that can induce instability. This can be deduced for systems with a variational structure ${ }^{2}$ by representing the potential energy with respect to all dynamically accessible configurations: There are more possible configurations with less potential energy for less-constrained equilibrium states and, therefore, more possible modes of instability ${ }^{41,45,46}$. Applications of these concepts to contact-line constraints, which are of interest to contact-drop dispensing, have been discussed in recent reviews ${ }^{47-49}$.

By definition, $\mathrm{CAH}$ is a relationship between the surface wettability and contact-line constraint on non-ideal surfaces and, thus, is expected to influ-

\footnotetext{
${ }^{2} \mathrm{~A}$ system with dynamics that are describable by a potential energy functional (e.g., conservative systems).
} 


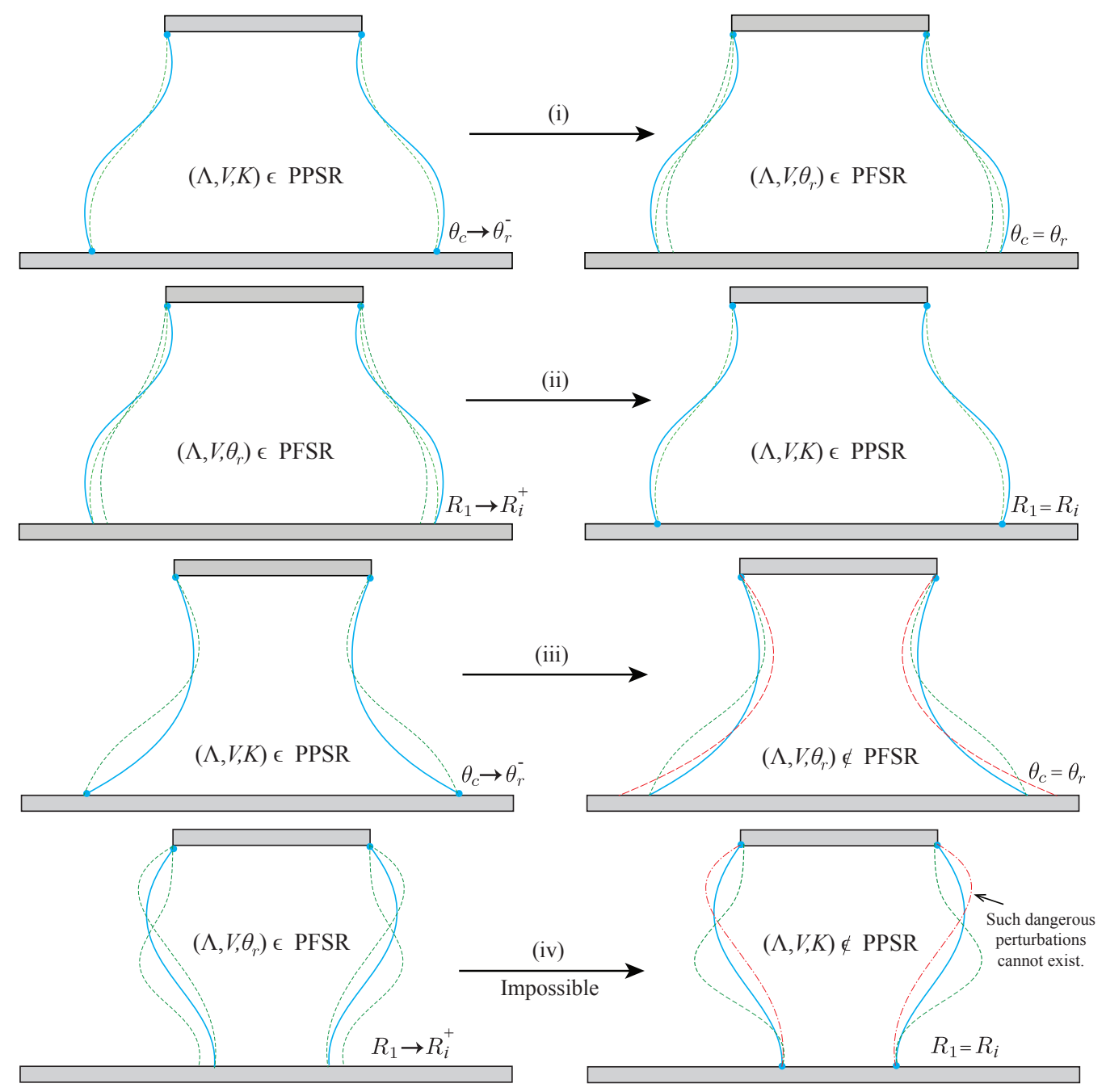

Figure 4: Pinned-to-free contact-line transition scenarios to be examined for stability loss of liquid bridges on surfaces with $\mathrm{CAH}$. Pinned-to-free transition arises when $\theta_{c}$ reaches $\theta_{r}$ as the bridge is stretched $\left(\theta_{c} \rightarrow \theta_{r}^{-}\right)$, whereas free-topinned transition occurs when a receding contact line is trapped by a circular indentation of radius $R_{i}$ centered on the symmetry axis $\left(R_{1} \rightarrow R_{i}^{+}\right)$. The meridian curve, $\Lambda, V, R_{1}$, and $\theta_{c}$ are identical before (left) and after (right) the transition. The stability region with respect to pinned-pinned (PPSR) and pinned-free (PFSR) perturbations are defined in $(\Lambda, V, K)$ and $\left(\Lambda, V, \theta_{c}\right)$ spaces. Filled circles, solid lines, short-dashed lines, and long-dashed lines, respectively, represent pinned contact lines, equilibrium meridian curves, safe perturbations, and dangerous perturbations. The dashed-dotted line shows a hypothetically dangerous, but physically impossible, perturbation. 
ence the stability of liquid bridges. It refers to a range of contact angles for which the contact line remains pinned. The contact line recedes (advances) if the contact angle approaches the receding (advancing) contact angle from below (above). Therefore, for a given drop volume and contact angle, one naturally expects that stability is lost at the stability limits of either pinnedpinned or pinned-free bridges, depending on the value of $\theta_{c}$ relative to $\theta_{r}$ and $\theta_{a}$. By contrast, the contact line is always free on ideal surfaces $^{3}$, where only the stability limits of pinned-free bridges are relevant.

To better understand how CAH can complicate contact-drop dispensing, consider stretching a liquid bridge spanning a needle and a non-ideal, homogeneous surface ${ }^{4}$, such as that shown in Fig. 2, where we seek to predict the breakup height $h_{b}$ for a given $v$ and $\theta_{r}\left(R_{1}\right)$ when the contact line on the surface is free (pinned). Suppose that the upper contact line ( $\bar{\ell}$ in Fig. 1) remains pinned to the needle edge at all times, while the lower contact line ( $\ell$ in Fig. 1) can be pinned or free depending on the contact-line position and contact angle. The contact line $\ell$ may also undergo pinned-to-free or free-to-pinned transitions during stretching. If $\theta_{c}<\theta_{r}$ at the beginning, the contact line remains pinned at the initial radius $R_{1,0}$, while $\theta_{c}$ increases (approaching $\theta_{r}$ from below) as the bridge is stretched from its initial state. Here, the bridge may reach its stability limit before $\theta_{c}$ reaches $\theta_{r}$, in which case $R_{1}=R_{1,0}$ and $h_{b}$ is estimated by the slender limit with respect to pinned-pinned perturbations. On the other hand, $\theta_{c}$ could reach $\theta_{r}$ from below $\left(\theta_{c} \rightarrow \theta_{r}^{-}\right)$before the stability limit is reached, in which case the bridge undergoes a pinned-to-free contact-line transition.

A transition in the reversed direction is also possible, for example if $\theta_{r}$ becomes larger than $\theta_{c}$ as a free contact line recedes on a heterogeneous surface during stretching. However, on a homogeneous surface (our assumption in this section), a free-to-pinned contact-line transition can only arise when $\ell$ is trapped by a sharp-edged circular indentation of radius $R_{i}<R_{1,0}$ with a center on the bridge symmetry axis. If $\theta_{c}=\theta_{r}$ at the beginning, the contact line recedes from its initial position at $R_{1,0}$ (approaching $R_{i}$ from above), while $\theta_{c}$ remains fixed at $\theta_{r}$. If a critical state is not reached as $R_{1}$ approaches $R_{i}$, then the bridge undergoes a free-to-pinned contact-line transition when $R_{1} \rightarrow R_{i}^{+}$.

Identifying the forgoing transitions alone is not always sufficient to predict

\footnotetext{
${ }^{3} \mathrm{An}$ ideal surface is defined as a surface without CAH.

${ }^{4} \mathrm{~A}$ surface with constant receding contact angle $\theta_{r}=$ const.
} 
$h_{b}$, because the state after the transition is not guaranteed to be experimentally realizable. One also needs to consider the interface stability after the transition, which, given that the interface must be stable before the transition for it to be of practical relevance, leads to four transition scenarios (see Fig. 4): (i) stable-pinned to stable-free, (ii) stable-free to stable-pinned, (iii) stable-pinned to unstable-free, and (iv) stable-free to unstable-pinned. The first two are trivial: the contact-line constraint in the slender limit determines $h_{b}$, irrespective of the history of the contact-line position. In the first, the bridge is stable before the transition, so $(\Lambda, V, K)$ belongs to the stability region with respect to pinned-pinned perturbations (PPSR) where $K$ corresponds to $R_{1}$ when $\theta_{c} \rightarrow \theta_{r}^{-}$. The bridge after the transition is also stable, so $\left(\Lambda, V, \theta_{r}\right)$ belongs to the stability region with respect to pinned-free perturbations (PFSR). In the second, $\left(\Lambda, V, \theta_{r}\right)$ belong to PFSR before the transition, and $(\Lambda, V, K)$ belongs to PPSR after the transition with $K=R_{i} / R_{0}$.

The third is a constraint-relaxing, destabilizing transition with nontrivial consequences, which we elaborate on in section 5.3. This scenario occurs for $(\Lambda, V, K)$ corresponding to states where the constraint at $\ell$ prevents dangerous pinned-free perturbations (those lowering $E$ ) from destabilizing the bridge. Once $\theta_{c} \rightarrow \theta_{r}^{-}$, this constraint is relaxed and the dangerous pinnedfree perturbations become admissible, causing stability loss. The fourth is a constraint-imposing, stabilizing transition. However, it is irrelevant to our study, because it cannot occur in practice. To clarify this point, suppose that there exist a dangerous pinned-pinned perturbation with a smaller $E$ than the equilibrium state. If such a perturbation existed, it would also be an admissible pinned-free perturbation for the state before the transition. Thus, there would be a perturbed state for the bridge before the transition with a smaller $E$ than in the equilibrium state, which would contradict the assumption that the state before the transition is stable.

\section{Results and discussion}

Results in this section are presented as follows: Section 5.1 demonstrates that, in a water-methanol-silicon oil system, adding SDS to the bath simulates drop dispensing on a hydrophobic surface and, thus, promotes mobility of the bridge-plate contact line. Section 5.2 experimentally validates the theoretical predictions of the stability limits with respect to pinned-free perturbations ${ }^{35}$. To avoid complications arising from the third scenario, high 
concentrations of SDS (10 $\left.\mathrm{g} \mathrm{l}^{-1}\right)$ are used to promote the pinned- to freecontact-line transition, such that the first scenario above always applies. Section 5.3 specifically focuses on the consequences of the third transition scenario on the slender limit and ensuing breakup dynamics.

\section{$5.1 \quad$ Surfactant effect}

As previously stated, for large drops, the contact angle $\theta_{c}$ remains smaller than the receding contact angle $\theta_{r}$ during stretching. Thus, the contact line is pinned to the coverslip at breakup. To assess the stability limits of liquid bridges with a free contact line over a wider range of drop volumes, the contact angle was reduced by adding SDS to the bath solution. The critical micelle concentration (CMC) of SDS in pure water at $25^{\circ} \mathrm{C}$ is $\approx$ $2.36 \mathrm{~g} \mathrm{l}^{-150}$. Note that equilibrium contact angles can vary on heterogeneous surfaces depending on the contact-line position. Consequently, the receding and advancing contact angles for the sessile drop can be sensitive to the drop volume. Therefore, we examine how the contact angle varies with the SDS concentration (within an order of magnitude of CMC) at various drop volumes to determine the surfactant concentration at which the contact angle is a minimum for all volumes. The contact angle was measured using the sessile-drop method ${ }^{51}$.

Figure 5 shows the surfactant effect on the contact angle. The contact angle decreases almost linearly around the CMC and below $\sim 6 \mathrm{~g} \mathrm{l}^{-1}$ for all drop volumes. At higher concentrations, the relationship is nonlinear. Nevertheless, the smallest value of the contact angle in the range $2-10 \mathrm{~g} \mathrm{l}^{-1}$ occurs at $10 \mathrm{~g} \mathrm{l}^{-1}$ for all volumes, except $5 \mu \mathrm{l}$. Moreover, the contact angle for larger drops is affected more by the surfactant at this concentration. Since larger drops tend to have a pinned contact line at breakup more often than smaller drops, only the stability-limit results for experiments where the SDS concentration is $10 \mathrm{~g} \mathrm{l}^{-1}$ are reported for all drop volumes. At this concentration, the bridge contact line with the coverslip was free at all drop volumes.

\subsection{Stability limits}

In this section, the theoretical predictions of the stability limits ${ }^{35}$ are tested experimentally. Since this paper only concerns the statics of stability loss and breakup, stability limits were ascertained through stepwise, quasi-static stretching and squeezing experiments. Thus, the Capillary, Weber, and 


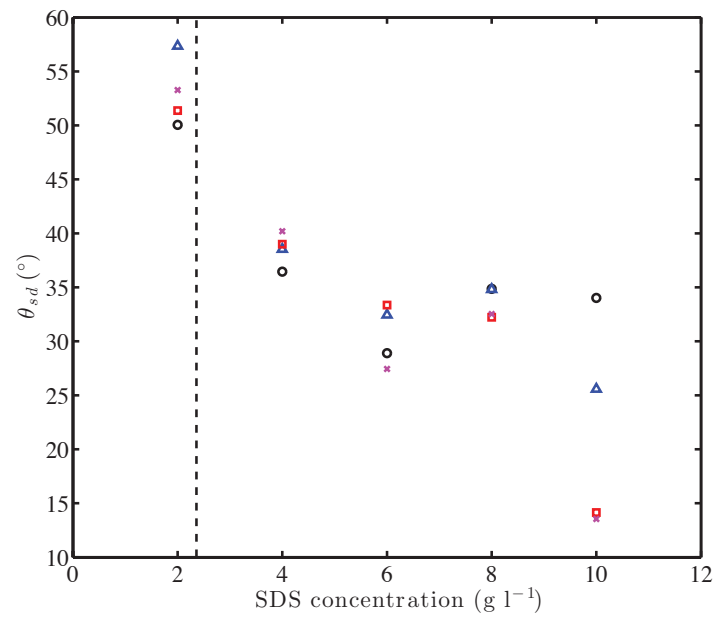

Figure 5: The surfactant-concentration effect on the sessile-drop contact angle $\theta_{s d}$ for drop volumes $5 \mu \mathrm{l}(\bigcirc), 10 \mu \mathrm{l}(\triangle), 15 \mu \mathrm{l}(\square), 20 \mu \mathrm{l}(\times)$. Dashed line identifies the critical micelle concentration for the surfactant in pure water at $25^{\circ} \mathrm{C}^{50}$.

Reynolds numbers are expected to be negligible. Stretching and squeezing experiments were performed on separate coverslips to eliminate contactline distortion when the rotund limit is reached and contact-angle variations due to surface contamination (arising from bridge squeezing) (see section 1). Therefore, position-dependent receding and advancing contact angles could be attributed solely to surface intrinsic heterogeneities. In all experiments, the contact line was either pinned or receding when stretching, and always advancing when squeezing. Therefore, when the contact line is free, the receding contact angle $\theta_{r}$ is the relevant contact angle in stretching, and the advancing contact angle $\theta_{a}$ is the relevant contact angle in squeezing. This implies that the slender limit must be calculated for $\theta_{r}$ at which the bridge loses stability and compared with the corresponding bridge height from experiments. Similarly, the rotund limit must be calculated for $\theta_{a}$ at which the bridge bulges non-axisymmetrically and compared with the corresponding bridge height from experiments.

Figure 6 shows an image sequence during stretching and squeezing of a $5 \mu \mathrm{l}$ drop. Here, no surfactant was added to the bath, and the contact line was free during stretching and squeezing. The receding contact angle at the 

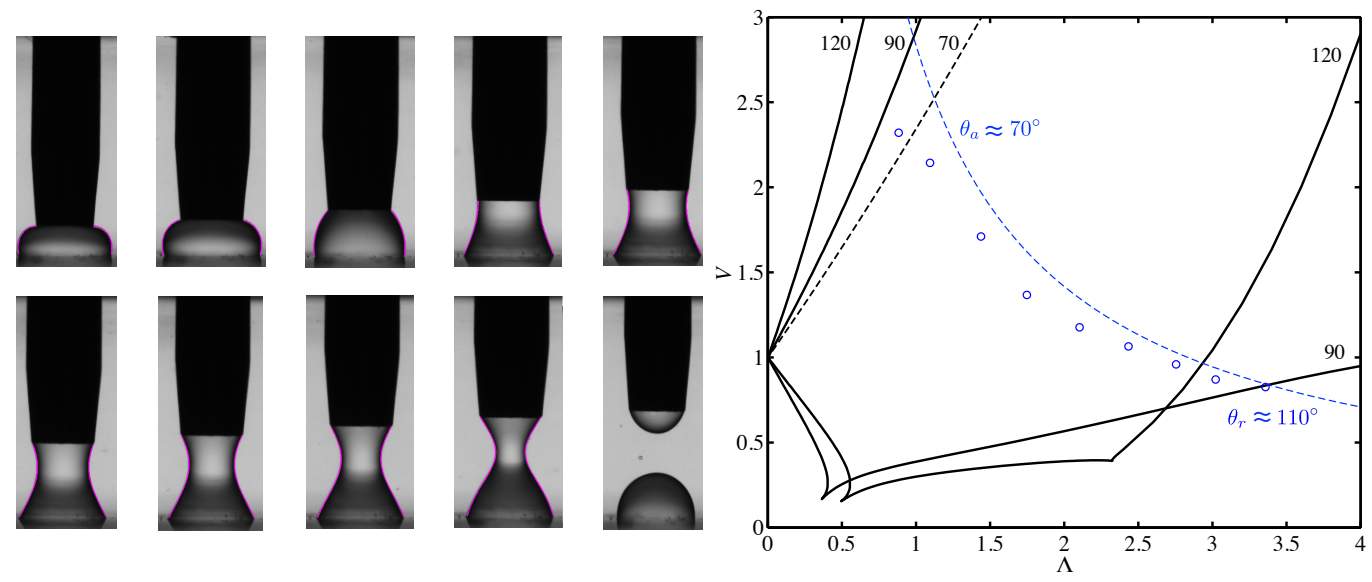

Figure 6: (left) An image sequence of the bridge evolution corresponding to the data points. (right) Comparison of the theoretical prediction and experimental measurement of the stability limits with drop volume $v_{i} \approx 5 \mu \mathrm{l}$, receding contact angle $\theta_{r} \approx 110^{\circ}$, advancing contact angle $\theta_{a} \approx 70^{\circ}$, and without surfactant. Dashed blue and black lines respectively indicate the constant- $v$ isocontour at $v=5 \mu \mathrm{l}$ and the maximum-volume stability limit estimated by Eq. (27) at $\theta_{c}=70^{\circ}$. Labels denote the contact angle in degrees.

slender limit was measured $\theta_{r} \approx 110^{\circ}$; thus, the corresponding data point (far right) in the stability diagram is expected to fall between the lower boundary of the stability region ${ }^{35}$ for $\theta_{c}=90^{\circ}$ and $120^{\circ}$, as demonstrated in Fig. 6 . The advancing contact angle at the rotund limit was measured $\theta_{a} \approx 70^{\circ}$; here, the minimum-slenderness stability limit is estimated by Eq. (27) and is then compared to the measured value. As shown in Fig. 6, experimental measurements of the stability limits are in good agreement with the theoretical predictions of Akbari et al. ${ }^{35}$. Note that the data points in Fig. 6, indicating the volume $v$ of the liquid between the planes $z=z\left(s_{1}\right)$ and $z=z\left(s_{0}\right)$ (see Fig. 1) as measured by image analysis ${ }^{5}$, generally deviate more from the constant- $v$ isocontour (dashed line) corresponding to the initial deposited-drop volume $v_{i}$ when the bridge is more squeezed because of the hollow needle: The more the bridge is squeezed, the more liquid is driven into the needle, so $v$ underestimates $v_{i}$ more at smaller $\Lambda$. Note also that

\footnotetext{
${ }^{5}$ Theoretically, at fixed bridge volume $v$, the scaled volume $v^{*}$ remains fixed, while the cylindrical volume varies with the slenderness as $V=4 v^{*} /(3 \Lambda)$ (see the definitions of $v^{*}$ and $V$ in section 2). Accordingly, the dashed line in Fig. 6 corresponds to $V=4 v_{i}^{*} /(3 \Lambda)$.
} 

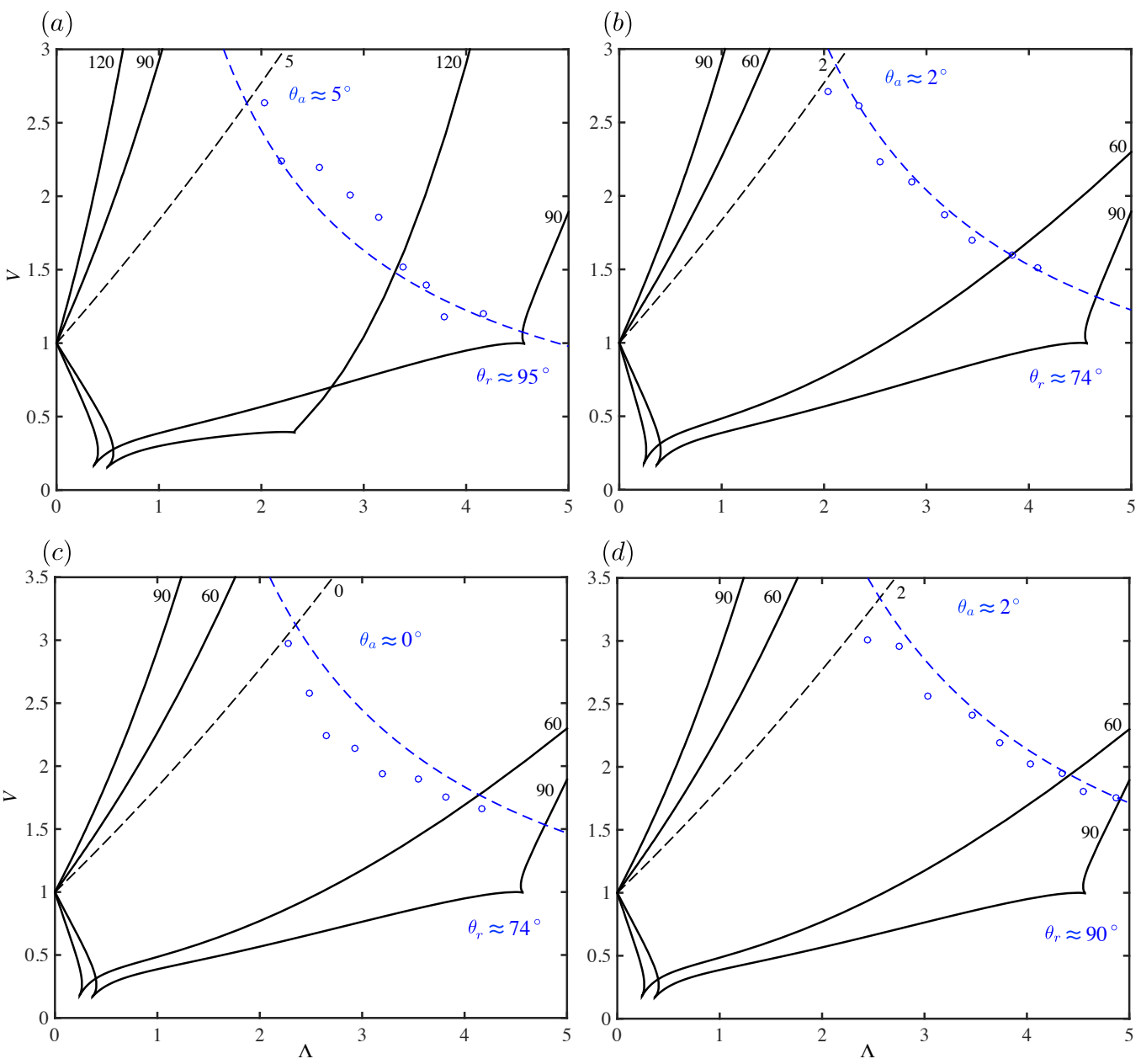

Figure 7: Same as Fig. 6, but with SDS concentration $10 \mathrm{~g} \mathrm{l}^{-1}$ at drop volumes (a) $v_{i}=10 \mu \mathrm{l}$, (b) $v_{i}=12.5 \mu \mathrm{l}$, (c) $v_{i}=15 \mu \mathrm{l}$, and (d) $v_{i}=17.5 \mu \mathrm{l}$. 
squeezing and stretching experiments were stepwise and quasi-static. Thus, although the volume obtained from image analysis differs from the initial volume for each data point, the bridge is in equilibrium with a fixed volume at each step; consequently, it is subject to volume preserving perturbations and stability is lost at the constant-volume slender limit upon stretching.

Stretching and squeezing experiments were conducted in the range $v=10$ $17.5 \mu \mathrm{l}$ with SDS added to the bath. At $10 \mathrm{~g} \mathrm{l}^{-1} \mathrm{SDS}$, the advancing and receding contact angles decreased to $\theta_{a} \approx 0-5^{\circ}$ and $\theta_{r} \approx 75-95^{\circ}$. Reasonable agreement was observed between the experimental measurements of the stability limits and theoretical predictions of Akbari et al. ${ }^{35}$ (see Fig. 7).

We conclude this section by emphasizing practical challenges that free contact lines may pose for measuring stability limits, especially on non-ideal surfaces where imperfections can cause uncertainty in the contact-line position and contact angle. When the contact lines are free, measuring the breakup height $h_{b}$, for example, at fixed $v$ and $\theta_{r}$ is of interest. Here, one needs to control surface wettability ${ }^{6}$ such that the bridge breaks while maintaining the desired $\theta_{r}$ and a free contact line. However, this is challenging to accomplish on highly heterogeneous surfaces, since $\theta_{r}$ varies during stretching according to the contact-line position, which, as discussed in section 4, can cause transitions in the contact-line constraint. Thus, ensuring a consistent breakup on heterogeneous surfaces at a fixed $\theta_{r}$ by controlling SDS concentration and bridge volume through repetitive measurements is difficult. In particular, this complicates the interpretation of error bars (normally regarded as uncertainty bounds in measurements that are only affected by random errors), because averages and variances of multiple measurements of the breakup height on heterogeneous surfaces represent a mixed effect of random errors and variations in local surface characteristics. Thus, the average breakup height $\bar{h}_{b}$ at the average receding contact angle $\bar{\theta}_{r}$ cannot necessarily be regarded as a valid measure of $h_{b}$ at $\theta_{r}$ and, consequently, is not comparable with theoretical values. Since the surfaces of the plastic coverslips in our experiments were highly heterogeneous, the purpose of this section was not to report the stability limits and associated variances for a given bridge

\footnotetext{
${ }^{6}$ Note that $\theta_{r}$ cannot be directly controlled. In practice, it can be altered by adjusting interfacial tensions (changing SDS concentration in our experiments) or using coverslips with different surface characteristics. This indirect control of $\theta_{r}$ is the primary complicating factor for free contact lines. In contrast, liquid bridges with pinned contact lines can be experimentally realized by squeezing and stretching a drop between two sharp-edged discs, where the contact line position is directly controlled by the diameter of the discs.
} 

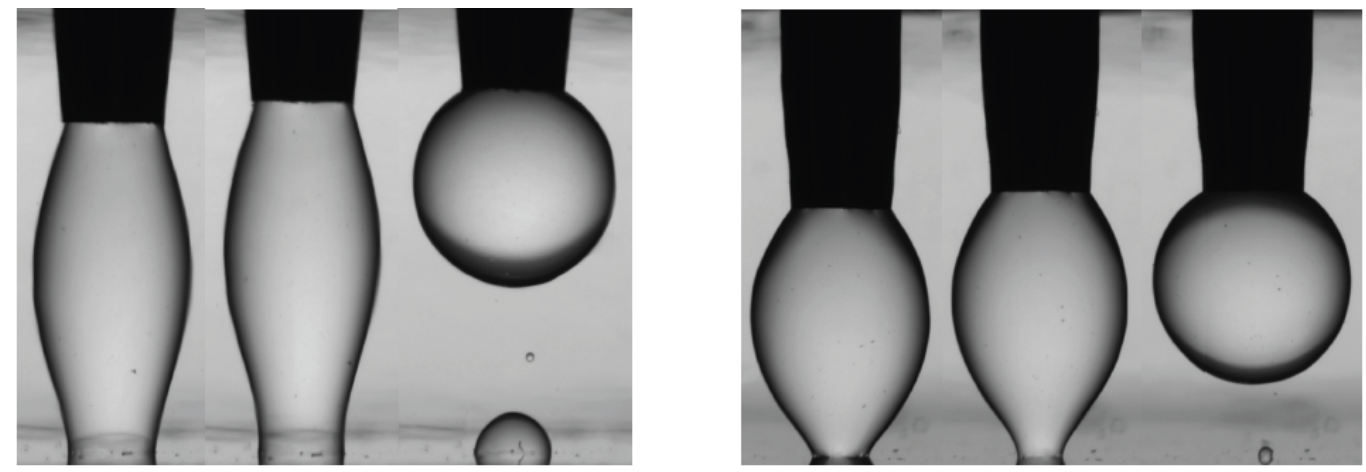

Figure 8: Contact-line effect on the breakup height of liquid bridges. Stretching a $20 \mu \mathrm{l}$ drop with $10 \mathrm{~g} \mathrm{l}^{-1}$ SDS in the bath, producing a pinned contact line with $\theta_{c} \approx 84^{\circ}$ (left) and a free contact line with $\theta_{c} \approx 81^{\circ}$ (right) at the slender limit.

volume and SDS concentration. Rather, we reported the slender limit for a given volume and a receding contact angle obtained from the bridge image before stability loss.

\subsection{Contact-angle hysteresis effect}

Liquid bridges with a free contact line lose stability at a shorter height than they would if the contact line was pinned because of the destabilizing effect of free contact lines ${ }^{35}$. In this section, we first demonstrate this effect, which is a central aspect of liquid-bridge breakup on surfaces with $\mathrm{CAH}$, in a qualitative experiment. Then, we study this experiment in more detail and examine possible consequences of stability loss according to the third scenario discussed in section 4 .

Figure 8 shows the effect of the contact-line constraint on the slender limit. Here, experiments were conducted on two coverslips using a $20 \mu \mathrm{l}$ drop with $10 \mathrm{~g} \mathrm{l}^{-1}$ SDS. Stretching and squeezing experiments were performed on separate coverslips for the left bridge, similarly to the procedure in section 5.2, so there was no sharp variation in the contact-line radius between squeezing and stretching since the contact line expanded and contracted, respectively, from an initial radius that was almost the same on both coverslips. However, squeezing and stretching were consecutively performed on the same coverslip for the right bridge. Here, upon stretching, the contact 
line retreated on a surface that had been covered by the silicon oil during squeezing, which affected $\gamma_{s g}$ and $\theta_{r}$ compared to the left bridge. This change of procedure allowed to control the contact-line constraint at breakup and to realize stability loss with respect to pinned-pinned and pinned-free perturbations for the same bridge volume and surfactant concentration. The contact line was pinned on both coverslips during the initial phase of stretching where $\theta_{c}$ increased with $\Lambda$ at fixed $K$. While the bridges were approaching breakup, $\theta_{c}$ remained below $\theta_{r}$, and the contact line remained pinned until breakup on one (Fig. 8, left panel), whereas $\theta_{c}$ reached $\theta_{r}$ before breakup on the other (Fig. 8, right panel) whereupon the contact line started receding; consequently, the contact line was pinned on the former and free on the latter when stability was lost. The slendernesses at the slender limit were measured $\Lambda_{b} \approx 5.99$ and $\Lambda_{b} \approx 4.94$, respectively. This $\approx 20 \%$ decrease in the breakup height reflects the destabilizing effect of a free contact line, which is consistent with the predictions of Akbari et al. ${ }^{52}$ for catenoidal and cylindrical liquid bridges. Chen et al. ${ }^{53}$ also reported experimental observations where constraint relaxation at the contact lines resulted in stability loss at shorter heights for liquid bridges of the same volume spanning two supports with close wettabilities.

Figure 9 quantitatively compares the contact-line constraint at breakup between the two liquid bridges in Fig. 8 by showing variations of the contactline radius $R_{1}$ with the bridge height. Clearly, as breakup is approached, the contact-line motion of the right bridge in Fig. 8 is more appreciable than the left bridge, indicating that the contact line on the left coverslip is more constrained than that on the right.

Hereafter, we focus on the data for the bridge with a free contact line at breakup (circles) in Fig. 9 and examine how breakup is affected when stability loss coincides with change in the contact-line constraint (i.e., during a pinnedto free-contact-line transition). Figure 10 shows the bridge-evolution images, comparing the theoretical prediction of the contact-line radius and measured values. Reasonable agreement is observed between the measurements and theoretical predictions. The last image $H$ was acquired after stability loss and before breakup, so it does not correspond to a stable equilibrium state. At this point, the bridge has already started accelerating towards rupture with the dynamic contact angle increasing from $\theta_{r} \approx 75^{\circ}$ at $P_{5}$ to $\theta_{r} \approx 81^{\circ}$ at $H$. This point is still away from breakup and, therefore, expected to follow an unstable solution of the Young-Laplace equation on an unstable equilibrium branch (see $\mathrm{H}$ in the branching diagram of Fig. 12). 


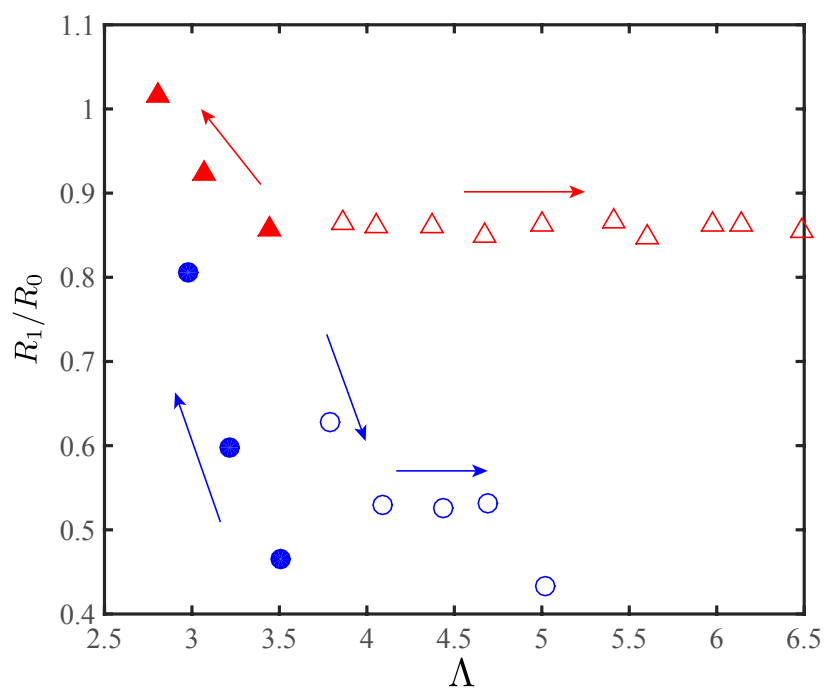

Figure 9: Squeezing (filled symbols) and stretching (open symbols) of a $20 \mu \mathrm{l}$ drop with $10 \mathrm{~g} \mathrm{l}^{-1} \mathrm{SDS}$, corresponding to the experiments shown in Fig. 8. The radius of the meniscus contact line $R_{1}$ versus slenderness are plotted when the bridge contact-line on the coverslip at breakup is free $(\bigcirc$, right panel in Fig. 8) and pinned ( $\triangle$, left panel in Fig. 8).

Note that the coverslip in this experiment exhibited an advancing contact angle $\theta_{a} \approx 3^{\circ}$ and two distinct receding contact angles $\theta_{r 1} \approx 30^{\circ}$ and $\theta_{r 2} \approx$ $75^{\circ}$. This can be attributed to changes in the surface tensions associated with the coverslip $\left(\gamma_{s g}\right.$ and $\left.\gamma_{s l}\right)$ when the contact line retreated on a surface that had already been covered by silicon oil.

The contact line was free during the entire squeezing experiment, and the contact angle remained almost fixed at $\theta_{c}=\theta_{a}$. However, during stretching, the contact line was pinned $(K \approx 0.7699)$ when $\theta_{a}<\theta_{c}<\theta_{r 1}$, free while the contact angle was almost fixed at $\theta_{c}=\theta_{r 1}$, and pinned $(K \approx 0.5271)$ when $\theta_{r 1}<\theta_{c}<\theta_{r 2}$. The trajectory $P_{1} P_{2}$ indicates the equilibrium solution corresponding to the squeezing, whereas $P_{2} P_{3}, P_{3} P_{4}$, and $P_{4} P_{5}$ correspond to the foregoing pinned-pinned, pinned-free, and pinned-pinned regimes of the stretching experiment. The point $P_{5}$ corresponds to a state where $\theta_{c}=\theta_{r 2}$, indicating a transition from pinned-pinned bridges at $K=0.5271$ to pinnedfree bridges at $\theta_{c}=75^{\circ}$. Pinned-free bridges along $P_{3} P_{4}$ are not stretched beyond their stability limit (thin dashed-dotted line), neither are pinnedpinned bridges along $P_{4} P_{5}$ beyond their stability limits (thin dashed line). 

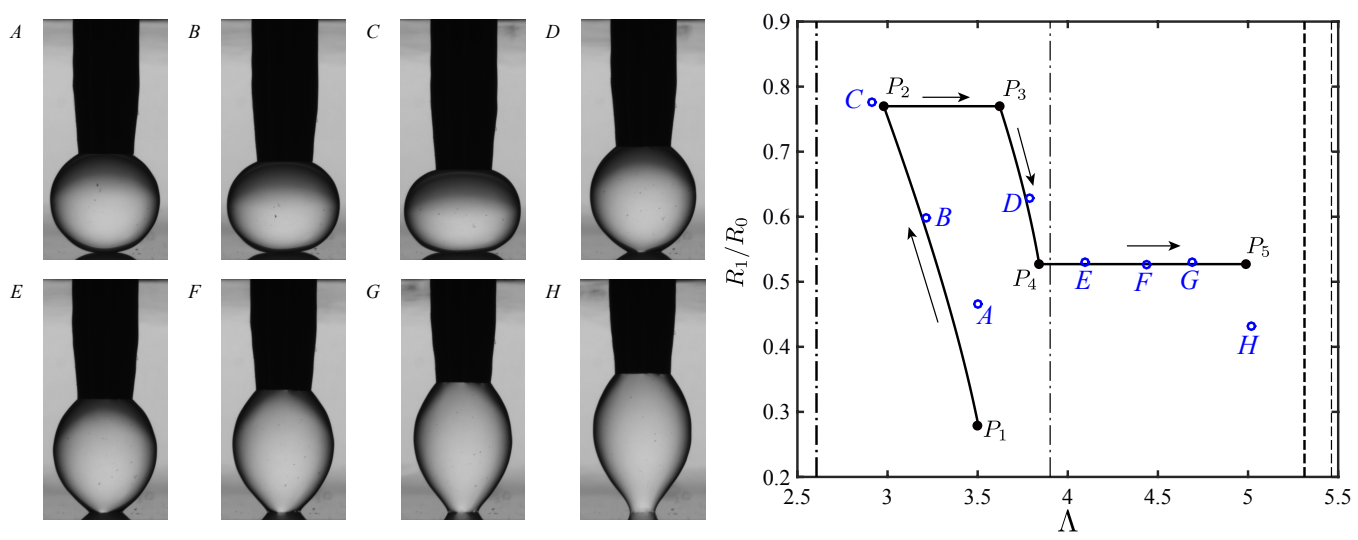

Figure 10: Comparison of experimental measurements (open circles) and theoretical predictions (solid lines) of the contact-line radius during the stretching and squeezing of a $20 \mu \mathrm{l}$ drop $\left(v_{i}^{*} \approx 7.171\right)$ on a substrate with an advancing $\left(\theta_{a} \approx 3^{\circ}\right)$ and two receding $\left(\theta_{r 1} \approx 30^{\circ}, \theta_{r 2} \approx 75^{\circ}\right)$ contact angle(s). Vertical lines indicate the minimum-high stability limit at $\theta_{c}=3^{\circ}$ (thick dashed-dotted) and maximum-high stability limits at $\theta_{c}=30^{\circ}$ (thin dasheddotted), $\theta_{c}=75^{\circ}$ (thick dashed), and $K=0.5271$ (thin dashed), where the contact line is free for the first three and pinned for the last. Equilibrium states are computed at fixed contact angle along $P_{1} P_{2}\left(\theta_{c}=5^{\circ}\right)$ and $P_{3} P_{4}$ $\left(\theta_{c}=30^{\circ}\right)$, and at fixed contact-line radius along $P_{2} P_{3}(K=0.7699)$ and $P_{4} P_{5}(K=0.5271)$.

Note that the slenderness at $P_{5}$ is also well below the pinned-free stability limit (thick dashed line) where the foregoing transition occurs. Therefore, stability is lost upon stretching at a state that is neither the pinned-pinned nor pinned-free stability limit.

To understand why stability is not lost at a critical state, we locate the stretching and squeezing trajectories of Fig. 10 on equilibrium branches in Figs. 11 and 12. Here, the bridge follows stable equilibrium branches along the entire $P_{1} P_{5}$ trajectory and before the transition at $P_{5}$. We note that the bridge lies on a stable branch with respect to pinned-pinned disturbance (see Fig. 12b) before the turning point. When $\theta_{c}$ reaches $\theta_{r 2}$ at $P_{5}$ (see Fig. 11c), the bridge is subjected to pinned-free disturbances. Although the slenderness is smaller than the maximum-slenderness stability limit, this state lies on an unstable branch, where the bridge loses stability to pinnedfree perturbations. Thus, in systems with $\mathrm{CAH}$, it is possible for liquid 

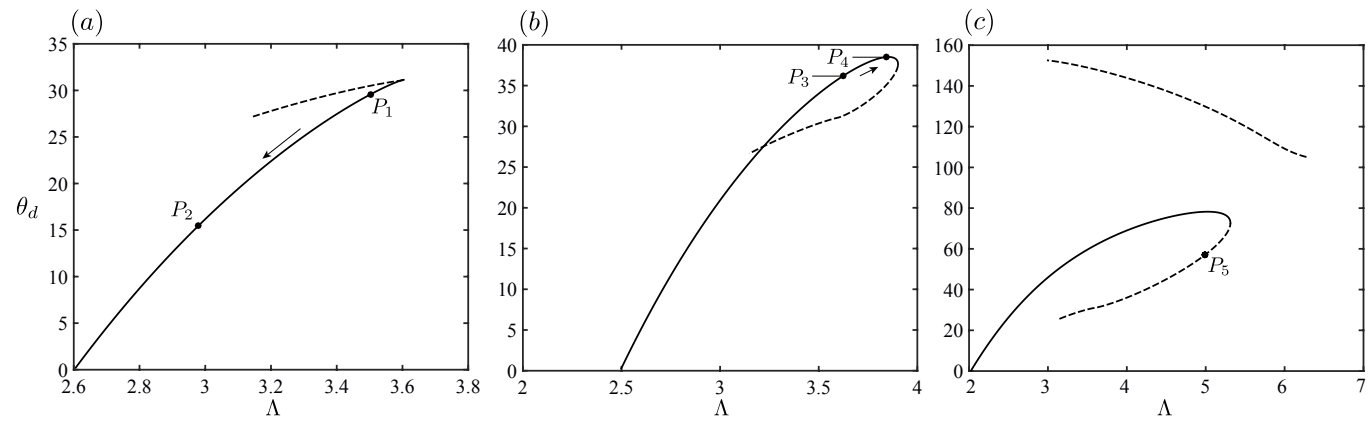

Figure 11: Free-contact line equilibrium branches of a fixed-volume $\left(v^{*}=\right.$ 7.171) liquid bridge, indicating stable (solid) and unstable (dashed) states at (a) $\theta_{c}=3^{\circ}$, (b) $\theta_{c}=30^{\circ}$, and (c) $\theta_{c}=75^{\circ}$. The terminal points $P_{1-5}$ are shown to identify the stability of equilibrium trajectories in Fig. 10.

bridges to break during a pinned-pinned to pinned-free transition rather than at a critical state. Accordingly, stability loss can be expedited by modulating CAH to induce breakup at a smaller height than an identical bridge would break if the contact line was perfectly free or perfectly pinned. This is a unique feature of surfaces with $\mathrm{CAH}$, because when the contact line is perfectly free or perfectly pinned, stability is always lost at a critical state. Conversely, stability loss can be delayed by adjusting CAH so that the contact line remains pinned during stretching, and the bridge breaks at a larger height than an identical bridge with a perfectly free contact line.

These observations could also have significant implications for the breakup dynamics and dispensed-drop volume. Recall, pinned-pinned liquid bridges are more stable than pinned-free ones. Consequently, the foregoing transition provides access to unstable states of pinned-free bridges that are far from critical sates. Before the transition, the contact line is pinned, and, depending on the bridge volume and receding contact angle, the bridge can be stretched to a state that is highly unstable to pinned-free perturbations. Once the receding contact angle is reached, the constraint at the contact line is relaxed, and the interface is exposed to a larger set of perturbations. This leads to a dramatic stability loss at a point that is not a critical state $\left(P_{5}\right.$ in Figs. $12 \mathrm{~b}$ and 11c). Note that the energy barrier (potential well) disappears at critical states, and the instability $\operatorname{margin}^{7}$ generally grows as an unstable

\footnotetext{
${ }^{7}$ The notion of the instability margin in this paper is the stability-margin counterpart of Slobozhanin et al. ${ }^{54}$ for unstable bridges. Note that liquid bridges break into several
} 

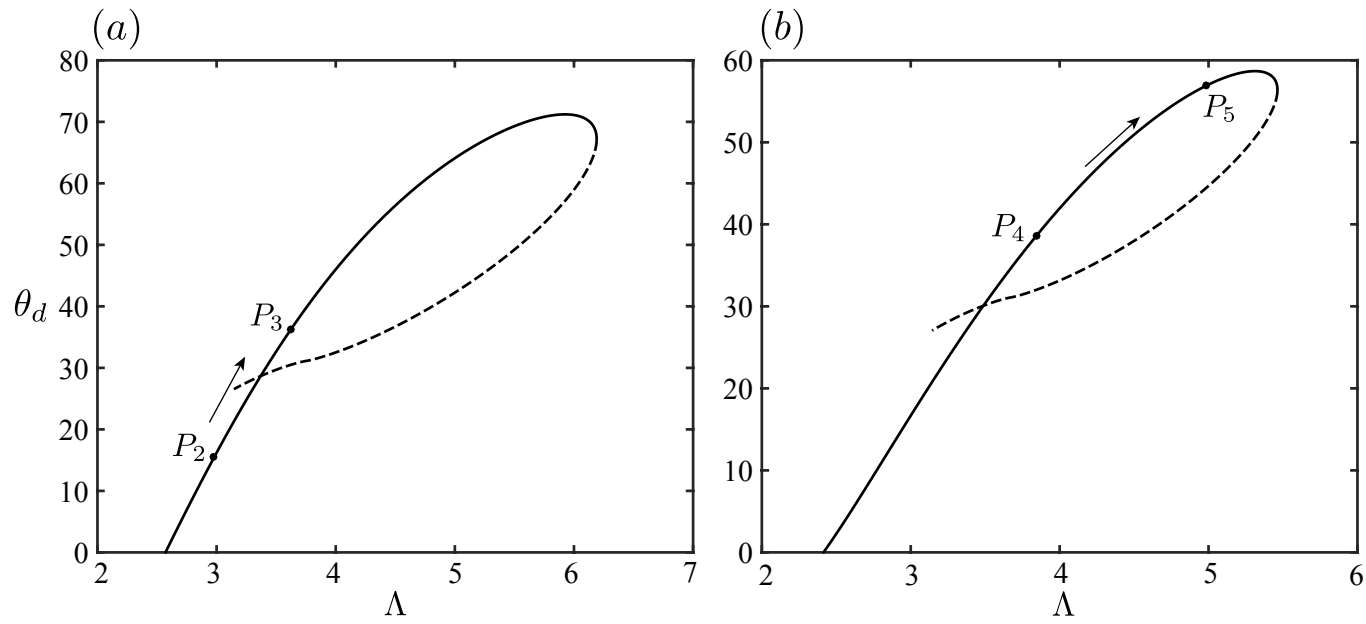

Figure 12: Pinned-contact line equilibrium branches of a fixed-volume $\left(v^{*}=\right.$ 7.171) liquid bridge, indicating stable (solid) and unstable (dashed) states at (a) $K=0.7699$ and (b) $K=0.5271$. The terminal points $P_{2-5}$ are shown to identify the stability of equilibrium trajectories in Fig. 10.

state moves farther away from its critical state along the respective equilibrium branch ${ }^{54,55}$. The potential energy of the instability margin can be transformed to kinetic energy upon stability loss, driving the near-singularity dynamics ${ }^{56}$.

An appreciable difference between the breakup dynamics upon stability loss at a critical state and at an unstable state away from its critical state is expected. At a critical state (e.g., turning point), the energy barrier disappears in the direction of the critical perturbation, so the interface accelerates in the same direction with a kinetic energy that is proportional to the disturbance magnitude. However, at an unstable state away from its critical state, the instability margin amplifies the critical perturbation, leading to more dramatic dynamics, which could impact the dispensed-drop size. As previously stated, Qian et al. ${ }^{24}$ reported small drop sizes in a pressure-controlled deposition due to fast dynamics near the contact line. Here, achieving a fast-receding contact line is assisted by the withdrawal of the liquid near

primary and satellite drops upon stability loss at the maximum-height stability limit ${ }^{19}$. Thus, the chain of drops arising upon breakup is the most stable (having the deepest potential well) state that is dynamically accessible to unstable bridges. Accordingly, the instability margin is defined as the potential-energy difference between the most stable and unstable states. 
the plate, which is less significant in the volume-controlled case ${ }^{25}$. However, breakup at an unstable state with a large instability margin could greatly influence the dynamics in volume-controlled deposition, potentially favouring smaller deposited drops. Further studies are required to examine the possibility of stability loss at unstable states with large instability margins, and their ensuing dynamics.

We further elaborate on the behaviour of the contact-line radius after stability loss by constructing the equilibrium branches at contact angles near $\theta_{r 2}$. The scaled form of the slender-jet approximation, commonly used in the literature to simplify near-pinchoff dynamics ${ }^{57-59}$, suggests that, except very close to the singularity where the capillary number $\mathrm{Ca} \sim O(1)$, the bridge profile can be reasonably approximated by the Young-Laplace equation. This approximation is expected-also experimentally shown by Qian and Breuer ${ }^{25}$ - to be accurate for volume-controlled stretching, since the liquid velocity inside the bridge is restricted by the volume constraint. Therefore, the bridge must evolve along unstable equilibrium branches at fixed volume after stability is lost. We apply this approximation as a guide to investigate the bridge evolution after stability loss and away from the pinch-off (e.g., bridge $H$ in Fig. 10) to provide a better understanding of the relationship between the receding contact angle and dispensed-drop volume.

Akbari et al. ${ }^{35}$ showed that, at fixed $\theta_{c}$, liquid bridges with a free contact line exhibit a transcritical bifurcation at a point along the lower boundary of the stability region. Transcritical bifurcations were represented by equilibrium branches at fixed $\theta_{c}$ and $\Lambda$ in pressure versus volume diagrams. Figure 13 shows equilibrium branches in the vicinity of a transcritical bifurcation in an $R_{1} / R_{0}$ versus $\Lambda$ diagram at fixed $v^{*}$ and $\theta_{c}$. As discussed for Figs. $12 \mathrm{~b}$ and 11c, the bridge after the pinned-pinned to pinned free transition at $P_{5}$ lies on an unstable segment of the primary branch at $\theta_{c}=75^{\circ}$. Upon stability loss, the dynamic contact angle ${ }^{8}$ increases from $\approx 75$ to $81^{\circ}$ when the bridge moves from the primary branch of $\theta_{c}=75^{\circ}$ at $P_{5}$ to the secondary branch of $\theta_{c}=81^{\circ}$ at $H$. Here, the opposite behaviour of $R_{1}$ with $\Lambda$ along stable and unstable branches is notable: the contact-line radius decreases (increases) during stretching along stable (unstable) branches, favouring small (large) drops. This implies that evolution upon stability loss at a fixed-contact-angle leads to larger drops; thus, to achieve smaller drops,

\footnotetext{
${ }^{8} \mathrm{~A}$ speed-dependent contact angle that deviates from the static receding contact angle after stability loss and before pinch-off due to high contact-line speeds.
} 


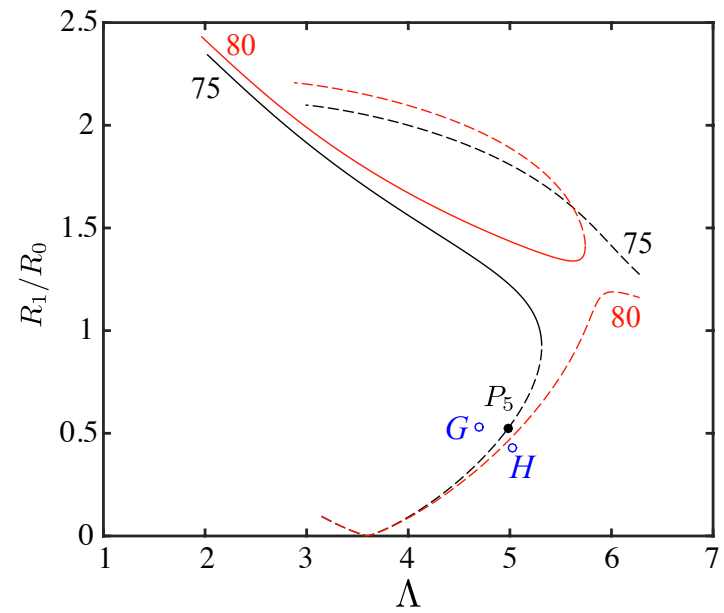

Figure 13: Free-contact line equilibrium branches of a fixed-volume $\left(v^{*}=\right.$ 7.171) liquid bridge in the vicinity of the transcritical bifurcation, indicating stable (solid) and unstable (dashed) states. Numeric labels denote the contact angle $\theta_{c}$ in degrees. Circles indicate the states at $G, H$, and $P_{5}$ as the bridge approaches breakup in Fig. 10.

the dynamic contact angle must increase following stability loss. Therefore, there are cases where a dynamic contact angle that deviates from the receding contact angle plays a key role in determining whether the contact line expands or contracts.

\section{Concluding remarks}

We have experimentally and theoretically studied the stability and breakup of weightless liquid bridges on surfaces with CAH. Experiments were performed in a Plateau tank where the effect of gravity was alleviated by density matching. To achieve free and pinned contact lines, the contact angle was adjusted by adding SDS to the bath. For liquid bridges with a free contact line, experimental measurements validated the theoretical predictions of the stability limits. At fixed volume, liquid bridges with a free contact line exhibit a smaller breakup height than those with a pinned contact line, demonstrating the destabilizing effect of a free contact line, as theoretically predicted by Akbari et al. ${ }^{35}$.

We examined the effect of $\mathrm{CAH}$ on the maximum-height stability limit, 
showing that the breakup height can not always be associated with the static stability limit. Depending on the drop volume and receding contact angle, liquid bridges may lose stability during the pinned-pinned to pinned-free transition at an unstable state away from its critical state. This has significant implications for the dynamics following stability loss. Unstable states that are far from critical states generally have large instability margins, which can transform to kinetic energy upon stability loss, having a significant impact on the breakup dynamics and dispensed-drop size. For example, the drop size in volume-controlled dispensing, which is not significantly affected by wettability, could be reduced if the advancing and receding contact angles are adjusted so that the bridge breaks during the pinned-to-free contact-line transition with faster dynamics. Constructing equilibrium branches in the vicinity of the receding contact angle revealed that a complex interplay between the dynamic contact angle (determined by its speed), receding contact angle when losing stability, and bridge volume determine whether the contact line is expanding or contracting following a loss of stability. Furthermore, we showed (by one example) that the contact-line motion upon stability loss at fixed contact angle can be advancing, thus disfavouring small-drop deposition.

More comprehensive computational investigations of the stability and breakup of liquid bridges will hopefully provide deeper insights into the relationship between the $\mathrm{CAH}$ and dispensed-drop size, and on the effectiveness of surface hydrophobization (to modulate the contact angle) for microdeposition.

\section{Acknowledgements}

Supported by the NSERC Innovative Green Wood Fibre Products Network (R.J.H.), and a McGill Engineering Doctoral Award (A.A.). The authors gratefully acknowledge Jessie Zhang performing experiments and image analysis.

\section{References}

[1] M. García Velarde, Physicochemical Hydrodynamics: Interfacial Phenomena, Springer, 1988, vol. 174. 
[2] K. Salaita, Y. Wang and C. A. Mirkin, Nat. Nanotechnol., 2007, 2, 145-155.

[3] F. Huo, Z. Zheng, G. Zheng, L. R. Giam, H. Zhang and C. A. Mirkin, Science, 2008, 321, 1658-1660.

[4] W. Shim, A. B. Braunschweig, X. Liao, J. Chai, J. K. Lim, G. Zheng and C. A. Mirkin, Nature, 2011, 469, 516-520.

[5] A. E. Cohen and L. Mahadevan, P. Natl. Acad. Sci. USA, 2003, 100, 12141-12146.

[6] H. Kim and L. Mahadevan, J. Fluid Mech., 2006, 548, 141-150.

[7] A. Boudaoud, J. Bico and B. Roman, Phys. Rev. E., 2007, 76, 060102.

[8] B. Pokroy, S. H. Kang, L. Mahadevan and J. Aizenberg, Science, 2009, 323, 237-240.

[9] C. H. Mastrangelo and C. H. Hsu, J. Microelectromech. S., 1993, 2, 33-43.

[10] H. Kwon, H. Kim, J. Puëll and L. Mahadevan, J. Appl. Phys., 2008, 103, 093519.

[11] D. Chandra and S. Yang, Langmuir, 2009, 25, 10430-10434.

[12] S. Farshid-Chini and A. Amirfazli, Langmuir, 2010, 26, 13707-13714.

[13] A. Akbari, R. J. Hill and T. G. M. van de Ven, Proc. R. Soc. A, 2015, 471, 20150184.

[14] A. Akbari, R. J. Hill and T. G. van de Ven, SIAM J. Appl. Math., 2016, 76, 87-109.

[15] J. A. F. Plateau, Statique expérimentale et théorique des liquides soumis aux seules forces moléculaires, Gauthier-Villars, 1873.

[16] L. Rayleigh, Proc. R. Soc. A, 1879, 29, 71-97.

[17] L. Rayleigh, Proc. R. Soc. A, 1879, 10, 4-13.

[18] I. Martínez and J. M. Perales, J. Cryst. Growth, 1986, 78, 369-378. 
[19] J. Meseguer, L. A. Slobozhanin and J. M. Perales, Adv. Space Res., 1995, 16, 5-14.

[20] S. Dodds, M. da Silveira Carvalho and S. Kumar, Phys. Fluids, 2009, 21, 092103.

[21] A. Lutfurakhmanov, G. K. Loken, D. L. Schulz and I. S. Akhatov, Appl. Phys. Lett., 2010, 97, 124107.

[22] H. Chen, T. Tang and A. Amirfazli, Soft Matter, 2014, 10, 2503-2507.

[23] R. D. Piner, J. Zhu, F. Xu, S. Hong and C. A. Mirkin, Science, 1999, 283, 661-663.

[24] B. Qian, M. Loureiro, D. A. Gagnon, A. Tripathi and K. S. Breuer, Phys. Rev. Lett., 2009, 102, 164502.

[25] B. Qian and K. S. Breuer, J. Fluid Mech., 2011, 666, 554-572.

[26] A. Sanz and I. Martinez, J. Colloid Interf. Sci., 1983, 93, 235-240.

[27] M. J. Russo and P. H. Steen, J. Colloid Interf. Sci., 1986, 113, $154-$ 163.

[28] L. A. Slobozhanin, J. I. D. Alexander and A. H. Resnick, Phys. Fluids, 1997, 9, 1893-1905.

[29] N. A. Bezdenejnykh, J. Meseguer and J. M. Perales, Phys. Fluids A, 1992, 4, 677-680.

[30] N. A. Bezdenejnykh, J. Meseguer and J. M. Perales, Phys. Fluids A, 1999, 11, 3181-3185.

[31] B. J. Lowry and P. H. Steen, Int. J. Multiphas. Flow, 1994, 20, 439-443.

[32] J. F. Joanny and P.-G. de Gennes, J Chem. Phys., 1984, 81, 552-562.

[33] H. Chen, A. Amirfazli and T. Tang, Langmuir, 2013, 29, 3310-3319.

[34] L. Gao and T. J. McCarthy, Langmuir, 2006, 22, 6234-6237.

[35] A. Akbari, R. J. Hill and T. G. M. van de Ven, Phys. Rev. E., 2015, 92, 022404. 
[36] H. Chen, T. Tang, H. Zhao, K.-Y. Law and A. Amirfazli, Soft Matter, 2016, 1998-2008.

[37] H. Chen, T. Tang and A. Amirfazli, Phys. Fluids, 2015, 27, 112102.

[38] H. Chen, T. Tang and A. Amirfazli, Langmuir, 2015, 31, 11470-11476.

[39] A. D. Myshkis, V. G. Babskii, N. D. Kopachevskii, L. A. Slobozhanin, A. D. Tyuptsov and R. S. Wadhwa, Low-gravity fluid mechanics, Springer-Verlag Berlin, 1987.

[40] D. Bonn, J. Eggers, J. Indekeu, J. Meunier and E. Rolley, Rev. Mod. Phys., 2009, 81, 739 .

[41] I. M. Gelfand and S. V. Fomin, Calculus of variations, Dover publications, 2000.

[42] A. W. Neumann, R. David and Y. Zuo, Applied surface thermodynamics, CRC Press, 2012.

[43] R. U. Seydel, Practical bifurcation and stability analysis, Springer, 2009.

[44] D. Marr and E. Hildreth, Proc. R. Soc. B, 1980, 207, 187-217.

[45] T. I. Vogel, SIAM J. Appl. Math., 1987, 47, 516-525.

[46] J. H. Maddocks, Arch. Ratio. Mech. An., 1987, 99, 301-328.

[47] J. H. Snoeijer and B. Andreotti, Annu. Rev. Fluid Mech., 2013, 45, 269-292.

[48] S. Kumar, Annu. Rev. Fluid Mech., 2015, 47, 67-94.

[49] J. Bostwick and P. Steen, Annu. Rev. Fluid Mech., 2015, 47, 539-568.

[50] P. Mukerjee and K. J. Mysels, Critical micelle concentrations of aqueous surfactant systems, Dtic document technical report, 1971.

[51] J. Bachmann, A. Ellies and K. H. Hartge, J. Hydrology, 2000, 231, 66-75.

[52] A. Akbari, R. J. Hill and T. G. M. van de Ven, SIAM J. Appl. Math., $2015,75,2110-2127$. 
[53] H. Chen, T. Tang and A. Amirfazli, Eur. Phys. J. Spec. Top., 2015, 224, 277-288.

[54] L. A. Slobozhanin, J. I. D. Alexander and V. D. Patel, Phys. Fluids, 2002, 14, 209-224.

[55] A. Myshkis, USSR Comput. Math. Math. Phys., 1965, 5, 193 - 201.

[56] J. Eggers, Rev. Mod. Phys., 1997, 69, 865.

[57] J. Eggers, Phys. Rev. Lett., 1993, 71, 3458-3460.

[58] J. Eggers and T. F. Dupont, J. Fluid Mech., 1994, 262, 205-221.

[59] S. Dodds, M. Carvalho and S. Kumar, Phys. Fluids, 2011, 23, 092101. 